\title{
Analysis of RTM extended images for VTI media
}

\author{
Vladimir Li ${ }^{1}$, llya Tsvankin ${ }^{1}$, and Tariq Alkhalifah ${ }^{2}$
}

\begin{abstract}
Extended images obtained from reverse time migration (RTM) contain information about the accuracy of the velocity field and subsurface illumination at different incidence angles. Here, we evaluate the influence of errors in the anisotropy parameters on the shape of the residual moveout (RMO) in P-wave RTM extended images for VTI (transversely isotropic with a vertical symmetry axis) media. Using the actual spatial distribution of the zero-dip NMO velocity $\left(V_{\mathrm{nmo}}\right)$, which could be approximately estimated by conventional techniques, we analyze the extended images obtained with distorted fields of the parameters $\eta$ and $\delta$. Differential semblance optimization (DSO) and stack-power estimates are employed to study the sensitivity of focusing to the anisotropy parameters. We also build angle gathers to facilitate interpretation of the shape of RMO in the extended images. The results show that the signature of $\eta$ is dip-dependent, whereas errors in $\delta$ cause defocusing only if that parameter is laterally varying. Hence, earlier results regarding the influence of $\eta$ and $\delta$ on reflection moveout and migration velocity analysis remain generally valid in the extended image space for complex media. The dependence of RMO on errors in the anisotropy parameters provides essential insights for anisotropic wavefield tomography using extended images.
\end{abstract}

\section{INTRODUCTION}

Reverse time migration (RTM) is a wavefield-based imaging technique that employs the two-way wave equation (Baysal et al., 1983; McMechan, 1983) and, therefore, can produce accurate images for complex models. RTM involves two main steps: reconstruction of the source and receiver wavefields and application of an imaging condition (Berkhout, 1982; Claerbout, 1985; Sava and Hill, 2009).
Wavefield reconstruction requires knowledge of the velocity model and source wavelet.

Optimally, wavefield reconstruction in anisotropic media should be performed by solving the elastic wave equation, which is considerably more computationally expensive compared to that for the acoustic problem. Another complicating factor in elastic wavefieldbased imaging is the cross-talk between the P- and S-wavefields, which produces false events in the resulting section. Alkhalifah $(1998,2000)$ derives a dispersion relation and a fourth-order wave equation for "acoustic" VTI media by setting the shear-wave symmetry-direction velocity $V_{S 0}$ to zero. The wave equation proposed by Alkhalifah (2000) can be split into a system of two second-order coupled equations, which further decreases computational cost (Fletcher et al., 2009; Fowler et al., 2010; Duveneck and Bakker, 2011; Zhang et al., 2011). These acoustic wave equations accurately describe P-wave kinematics in TI media but produce shear-wave "artifacts" caused by setting $V_{S 0}$ to zero (Grechka et al., 2004). Alkhalifah (2000) suggests eliminating these artifacts by placing sources and receivers in a purely isotropic or elliptical medium. Alternatively, one can use mixed-domain extrapolation for a pure P-mode to avoid generating S-waves (Fomel et al., 2013b).

An imaging condition yields information about reflectivity (the image) by matching the computed source and receiver wavefields, which is often done by cross-correlation. The conventional imaging condition is defined as the zero-lag value of the cross-correlation between the source and receiver wavefields (Claerbout, 1985). In contrast, the extended (general) imaging condition retains the temporal and spatial correlation lags in the output and, therefore, contains information about the directionality of the wavefield and angle-dependent reflector illumination. For example, one can obtain time-lag (Sava and Fomel, 2006) or space-lag (Rickett and Sava, 2002) extensions. For large-scale models, especially if both timeand space-lag extensions are produced, the cost of computation and storage of such images can be prohibitive. This problem is usually addressed by analyzing extended common-image gathers (CIGs) computed only at fixed horizontal coordinates in the model space.

\footnotetext{
Manuscript received by the Editor 15 July 2015; revised manuscript received 3 November 2015; published online 28 April 2016.

${ }^{1}$ Colorado School of Mines, Center for Wave Phenomena, Golden, Colorado, USA. E-mail: vli@mymail.mines.edu; ilya@ dix.mines.edu.

${ }^{2}$ King Abdullah University of Science and Technology, Thuwal, Saudi Arabia. E-mail: tariq.alkhalifah@kaust.edu.sa.

(C) 2016 Society of Exploration Geophysicists. All rights reserved.
} 
Both time-lag and space-lag CIGs can be converted into angle gathers (Sava and Fomel, 2003) to facilitate analysis and interpretation of residual moveout. Sava and Vasconcelos (2009) propose to generate multilag extensions only at sparse points in the model space, which makes the computations significantly more efficient, especially for 3D migration. These extended commonimage-point (CIP) gathers preserve the sensitivity to velocity errors and, therefore, are suitable for migration velocity analysis (MVA) (Yang and Sava, 2015). Optimal locations of extended CIPs can be selected automatically based on the properties of the conventional image (Cullison and Sava, 2011).

Wavefield tomography based on minimization of the residual moveout (RMO) in the extended image domain has recently attracted considerable attention in the literature. It is well known that imaging with an inaccurate velocity model results in suboptimal focusing of energy at the zero-lag location. This defocusing of energy is commonly quantified using differential semblance optimization (DSO) (Symes and Carazzone, 1991; Shen et al., 2003; Shen and Symes, 2008) or a measure of stack power (Chavent and Jacewitz, 1995; Soubaras and Gratacos, 2007).

Transversely isotropic models with a vertical or tilted symmetry axis (VTI or TTI) are widely used in depth imaging and typically provide improvements in event focusing and reflector positioning. In structurally complex areas (e.g., near salt bodies), where ray theory fails to accurately describe wave propagation, RTM yields superior images compared to ones obtained with ray-based techniques such as Kirchhoff migration. The main difficulty in anisotropic imaging is robust estimation of model parameters, and RTM can also increase the accuracy of model building in the presence of complex structures. Understanding the influence of the anisotropy parameters on the residual moveout in RTM extended images should provide useful insights for wavefield tomography.

P-wave kinematics in VTI media is controlled by the vertical velocity $V_{P 0}$ and Thomsen parameters $\epsilon$ and $\delta$ (Tsvankin, 2012). An alternative parameter set includes the normal-moveout velocity for a horizontal interface $\left[V_{\mathrm{nmo}}(0)=V_{P 0} \sqrt{1+2 \delta}\right]$, the anellipticity parameter $\eta=(\epsilon-\delta) /(1+2 \delta)$, and coefficient $\delta$. Alkhalifah and Tsvankin (1995) demonstrate that for a laterally homogeneous VTI medium above the target reflector, P-wave reflection moveout and time-domain processing depend on only two parameters $V_{\text {nmo }}(0)$ and $\eta$. In the absence of lateral heterogeneity, the parameter $\delta$ does not influence the moveout and is needed only for time-todepth conversion.

Using velocity-continuation operators, Alkhalifah and Fomel (2011) analyze the anisotropic response for poststack time imaging in homogeneous acoustic VTI media. They demonstrate that the residual response due to errors in the parameter $\eta$ has a predominantly linear ("V"-like) rather than hyperbolic shape. Sava and Alkhalifah (2012) use the coupled pseudo-acoustic equations proposed by Fowler et al. (2010) and analyze extended CIPs (Sava and Vasconcelos, 2009) obtained with inaccurate values of $\eta$ in VTI and TTI media. They consider reflections from a horizontal interface with a constant value of $\eta$ above it and $\delta$ set to zero. They conclude that an error in $\eta$ produces consistent "V"-shape defocusing in extended images regardless of the complexity of the $V_{\text {nmo }}(0)$-field. An understated value of $\eta$ causes defocusing with the apex pointing up (" $\Lambda$ "), while if $\eta$ is too large, the apex points down ("V"). The slope of the flanks of the residual moveout is controlled by the magnitude of the error in $\eta$. Li et al. (2014) propose an algorithm for VTI image-domain wavefield tomography based on minimizing defocusing in extended images.

Here, we study the anisotropy signature in RTM extended images for VTI models with dipping and curved interfaces and laterally varying $\delta$-fields. We start by discussing the defocusing in the extended image domain caused by inaccuracies in the velocity model and reviewing the dependence of $\mathrm{P}$-wave reflection moveout on the VTI parameters. Then, using coupled pseudo-acoustic equations for wavefield extrapolation, we study the defocusing in space-lag CIGs and extended CIPs caused by errors in $\eta$ and $\delta$ for several models of varying structural complexity. The DSO and stack-power objective functions are employed to quantify the defocusing and access the feasibility of parameter estimation in the image domain. We also compute and analyze angle gathers, which aid in interpreting the RMO observed in the extended images.

\section{THEORY}

\section{Extended image domain}

Using a general imaging condition, one can obtain image extensions in both space and time (Sava and Vasconcelos, 2011):

$$
I(\mathbf{x}, \boldsymbol{\lambda}, \tau)=\sum_{\text {shots }} \sum_{t} W_{s}(\mathbf{x}-\lambda, t-\tau) W_{r}(\mathbf{x}+\boldsymbol{\lambda}, t+\tau),
$$

where $I(\mathbf{x}, \boldsymbol{\lambda}, \tau)$ is the extended image, $W_{s}$ and $W_{r}$ denote the source and receiver wavefields (respectively), $\lambda$ is the space lag, and $\tau$ is the time lag.

Wavefields can be generally considered locally planar in the vicinity of each subsurface point. Therefore, extended images carry information about how an image point is illuminated by plane waves incident at different angles from different shot locations. In the absence of illumination problems, imaging with an accurate velocity model produces planar events that intersect at zero space and time lag. Hence, the energy in the resulting extended image after summation over shots is focused primarily at zero lag (Sava and Alkhalifah, 2012; Yang and Sava, 2015). This is a manifestation of the semblance principle (Sattlegger, 1975; Al-Yahya, 1989) stating that the position of an imaged reflector at any subsurface point does not depend on the illumination direction. Therefore, velocity errors lead to residual energy appearing at nonzero lags, and these residuals could be used to update the model.

The defocusing in extended images can be quantified, for example, with the differential semblance optimization (DSO) operator (Symes and Carazzone, 1991; Shen et al., 2003; Shen and Symes, 2008):

$$
J_{\mathrm{DSO}}=\left\|\lambda_{x} I\left(\mathbf{x}, \lambda_{x}\right)\right\|^{2}
$$

where $I\left(\mathbf{x}, \lambda_{x}\right)$ is the extended image. Equation 2 represents the DSO objective function where the horizontal space lag $\lambda_{x}$ plays the role of a penalty operator by eliminating energy at zero lag and amplifying energy at nonzero lags. A similar objective function can also be formulated using the time lag (Yang and Sava, 2011). Alternatively, one can use the stack-power operator to quantify the energy in the conventional (zero-lag) image (Chavent and Jacewitz, 1995; Soubaras and Gratacos, 2007):

$$
J_{\mathrm{ST}}=\left\|I\left(\mathbf{x}, \lambda_{x}=0\right)\right\|^{2} .
$$


In the absence of illumination problems, imaging with an accurate velocity model focuses energy primarily around the zero-lag location, which minimizes the DSO operator and maximizes stack power. The objective function based on DSO can be combined with stack power in migration velocity analysis to increase the accuracy of the inverted model (Shen and Symes, 2008; Weibull and Arntsen, 2013, 2014; Li et al., 2014).

\section{Angle-domain decomposition}

As mentioned above, extended images contain information about angle-dependent subsurface illumination. If this information can be reliably retrieved, it can facilitate interpretation of the residual moveout. Different algorithms have been proposed to extract reflectivity as a function of incidence angle at the reflector from time- and space-lag extended images (Sava and Fomel, 2003, Sava and Vlad, 2011; Sava and Alkhalifah, 2011). In anisotropic media, the estimated angles correspond to the phase rather than group (ray) direction (Sava and Alkhalifah, 2011, 2012). For a dipping interface beneath a VTI medium, the incidence and reflection phase angles are not equal to each other (Tsvankin, 2012), and extended images yield average opening angles (Biondi, 2007).

\section{$P$-wave kinematics and wavefield reconstruction in VTI media}

Alkhalifah and Tsvankin (1995) demonstrate that P-wave reflection moveout for a laterally homogeneous VTI medium above the target horizon is controlled by the zero-dip NMO velocity and parameter $\eta$. In the case of a horizontal VTI layer, $\eta$ controls the nonhyperbolic (long-offset) portion of the P-wave moveout $t(x)$ :

$$
t^{2}=t_{0}^{2}+\frac{x^{2}}{V_{\mathrm{nmo}}^{2}(0)}-\frac{2 \eta x^{4}}{V_{\mathrm{nmo}}^{2}(0)\left[t_{0}^{2} V_{\mathrm{nmo}}^{2}(0)+(1+2 \eta) x^{2}\right]} .
$$

With effective values of $V_{\mathrm{nmo}}(0)$ and $\eta$, equation 4 describes the Pwave reflection moveout for a stack of horizontal VTI layers above the target horizon.

For a dipping interface beneath a homogeneous VTI layer, the Pwave NMO velocity depends on both $V_{\text {nmo }}(0)$ and $\eta$ (Tsvankin, 2012):

$$
V_{\mathrm{nmo}}(p)=\frac{V_{\mathrm{nmo}}(0)}{\sqrt{1-g}}[1+(\epsilon-\delta) f(g)],
$$

where

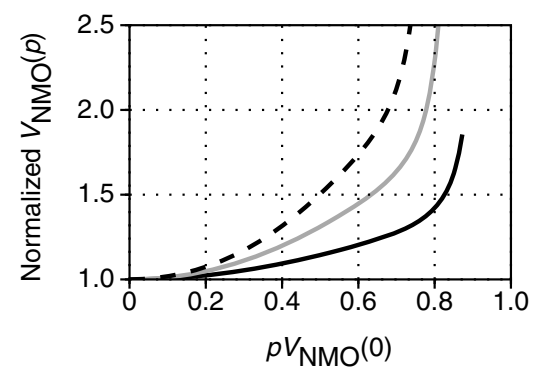

$p V_{\mathrm{NMO}}(0)$

$$
f(g) \equiv \frac{g\left(4 g^{2}-9 g+6\right)}{1-g}, \quad g \equiv p^{2} V_{\mathrm{nmo}}^{2}(0),
$$

and $p$ is the ray parameter of the zero-offset ray. Although equation 5 is a linearized (weak-anisotropy) approximation, the exact $V_{\text {nmo }}$ expressed as a function of $p$ is almost entirely controlled by $V_{\text {nmo }}(0)$ and $\eta$ (Figure 1). For VTI media with typical $\eta>0$, the NMO velocity increases much faster with dip compared to elliptical $(\epsilon=\delta)$ or purely isotropic models. If the dip reaches $25^{\circ}-30^{\circ}$, the NMO velocity becomes sufficiently sensitive to the parameter $\eta$ and can be used in time-domain DMO inversion (Anderson et al., 1996; Alkhalifah, 1996, 1997). For brevity, hereafter we denote $V_{\text {nmo }}(0)$ simply by $V_{\text {nmo. }}$.

If the VTI medium above the reflector is laterally homogeneous, the coefficient $\delta$ does not influence the moveout and, therefore, cannot be constrained by P-wave reflection traveltimes (Alkhalifah and Tsvankin, 1995; Tsvankin and Grechka, 2011; Tsvankin, 2012). Pwave moveout and time-domain processing still depend on just $V_{\text {nmo }}$ and $\eta$ even when these parameters vary laterally above the target horizon, but $\delta$ changes only with depth (Alkhalifah et al., 2001). However, if $\delta$ is laterally variable, P-wave traveltimes become sensitive to all three relevant parameters $-V_{\mathrm{nmo}}, \eta$, and $\delta$ (or $V_{P 0}, \epsilon$, and $\delta$ ) (Alkhalifah and Tsvankin, 1995; Tsvankin and Grechka, 2011; Tsvankin, 2012). Le Stunff et al. (2001) successfully constrain $V_{P 0}, \epsilon$, and $\delta$ for a relatively simple VTI model with an intermediate dipping interface using only $\mathrm{P}$-wave reflection traveltimes. In general, however, resolving all P-wave kinematic parameters requires additional information.

Inexpensive and kinematically accurate reconstruction of Pwavefields in TI models can be achieved by solving a system of two second-order coupled equations where the velocity $V_{S 0}$ is set to zero (Fletcher et al., 2009; Fowler et al., 2010; Duveneck and Bakker, 2011; Zhang et al., 2011). The 2D version of the formulation proposed by Fowler et al. (2010) can be written as:

$$
\begin{aligned}
& \frac{\partial^{2} p}{\partial t^{2}}=V_{\text {hor }}^{2} \frac{\partial^{2} p}{\partial x^{2}}+V_{P 0}^{2} \frac{\partial^{2} q}{\partial z^{2}} \\
& \frac{\partial^{2} q}{\partial t^{2}}=V_{\text {nmo }}^{2} \frac{\partial^{2} p}{\partial x^{2}}+V_{P 0}^{2} \frac{\partial^{2} q}{\partial z^{2}}
\end{aligned}
$$

where $V_{\text {hor }}=V_{\text {nmo }} \sqrt{1+2 \eta}=V_{P 0} \sqrt{1+2 \epsilon}$ is the P-wave horizontal velocity. Both the $p$-and $q$-components contain a wavefield with accurate P-wave kinematics and a shear-wave artifact with a diamond-shape wavefront caused by eliminating $V_{S 0}$. The existence of the artifact is explained by the fact that despite setting $V_{S 0}$ to zero, the SV-wave velocity does not vanish at all propagation angles. The false shear events in RTM images complicate evaluation of the

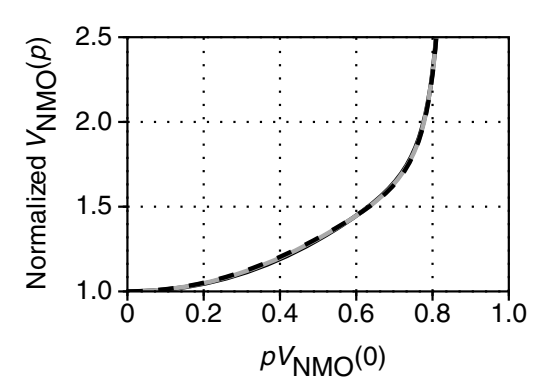

Figure 1. Exact P-wave NMO velocity calculated as a function of the ray parameter $p$ and normalized by the isotropic dependence $\left(V_{\text {nmo }}(0) / \sqrt{1-g}\right.$, see equation 5). Reflector dip ranges from $0^{\circ}$ to $70^{\circ}$. (a) Widely different models with the same $\eta=0.2: \quad \epsilon=0.1, \quad \delta=-0.071 \quad$ (solid), $\epsilon=0.2$, $\delta=0$ (gray), and $\epsilon=0.3, \delta=0.071$ (dashed). (b) Models with different $\eta: \eta=0.1$ (solid), $\eta=0.2$ (gray), and $\eta=0.3$ (dashed) (after Alkhalifah and Tsvankin, 1995, and Tsvankin, 2012). 
sensitivity of the focusing to model errors. One way to eliminate the $\mathrm{S}$-wave artifact is to place sources and receivers in a purely isotropic or elliptical $(\epsilon=\delta, \eta=0)$ medium (Alkhalifah, 2000; Duveneck et al., 2008).

\section{SYNTHETIC EXAMPLES}

Here, we analyze how the anisotropy parameters $\eta$ and $\delta$ influence the residual moveout in RTM extended images. The parameters $V_{\mathrm{nmo}}, \eta$, and $\delta$ are defined on a rectangular grid, and the density is assumed to be constant. For wavefield extrapolation, we use the finite-difference (FD) algorithm sfttifd2d in MADAGASCAR that solves the system in equation 7 . For all synthetic examples, the sources and receivers are located at the surface. The near-surface layer is taken to be isotropic to suppress the shear-wave artifact. We obtain space-lag and time-lag RTM extended images with different $\eta$ - and $\delta$ - fields, while using the actual $V_{\text {nmo }}$. To facilitate the interpretation of residual moveout, space-lag common-image gathers are also converted into angle-domain CIGs.

\section{Model 1}

In the first test, we evaluate the signature of $\eta$ for an interface beneath a homogeneous VTI layer. The reflector has a syncline shape with the dip of the flanks equal to $30^{\circ}$ (Figure 2). First, we

Figure 2. Model with a syncline beneath a homogeneous VTI medium: (a) $V_{\text {nmo }}$, (b) $\eta$ (in the VTI layer, $\eta=0.15$ ), and (c) $\delta$ (model 1). (d) Conventional RTM image obtained with the actual model. The vertical lines mark the locations of extended CIGs $(x=1.5,4.0,6.5 \mathrm{~km})$ used in the analysis.

a)

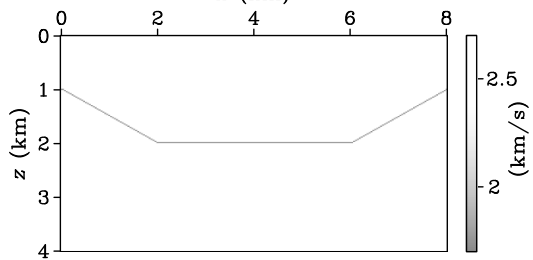

b)

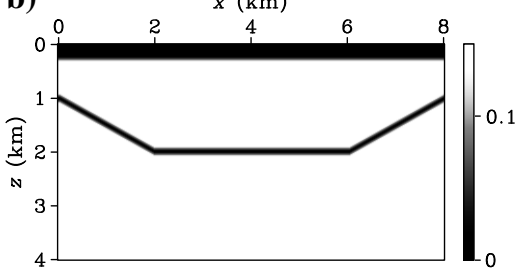

c)
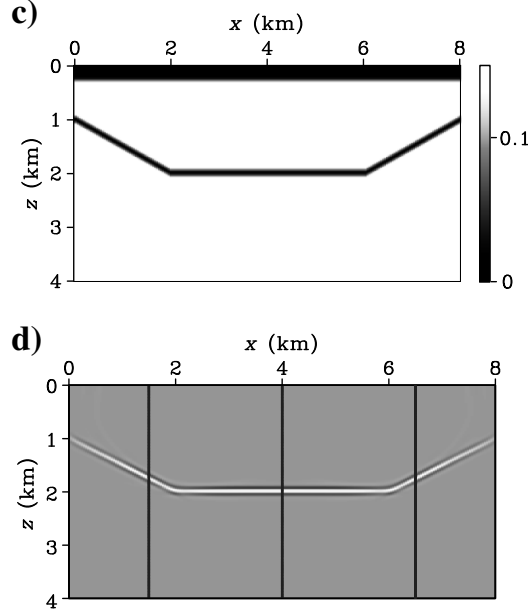

a)

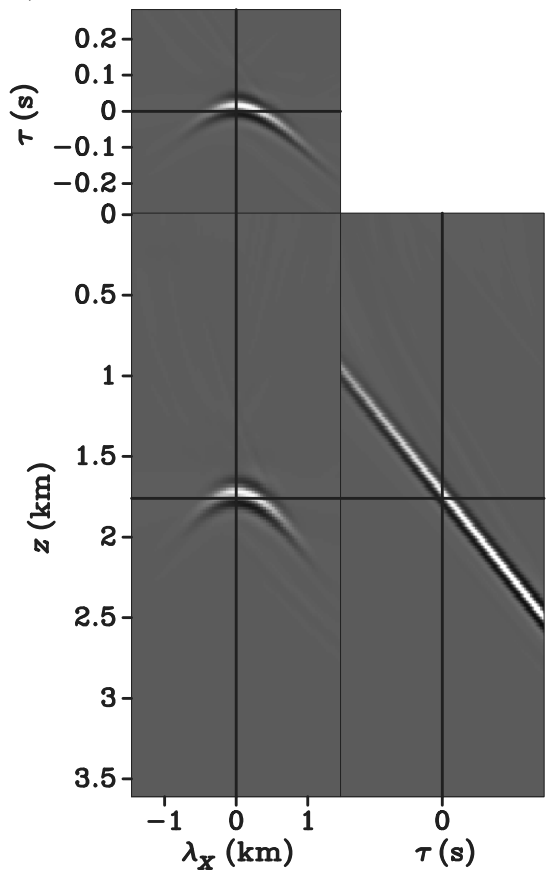

b)

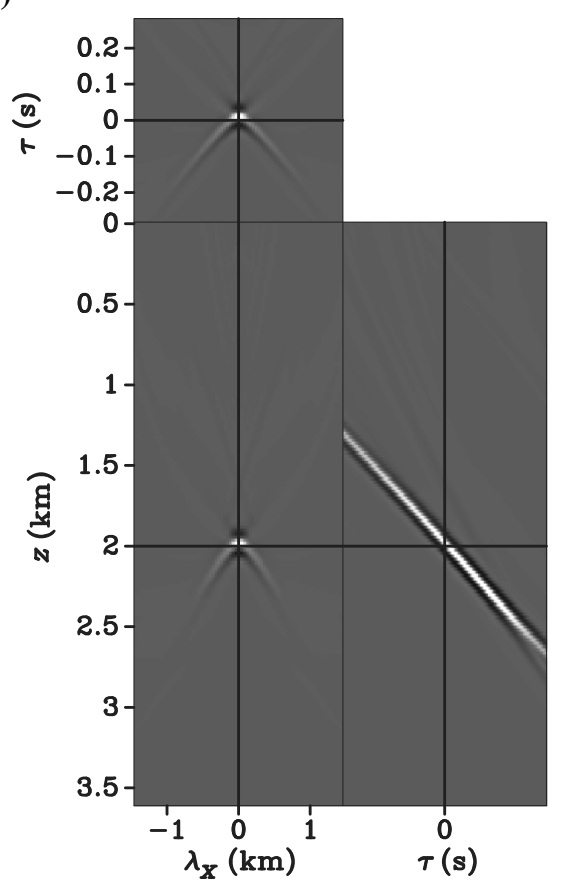

c)

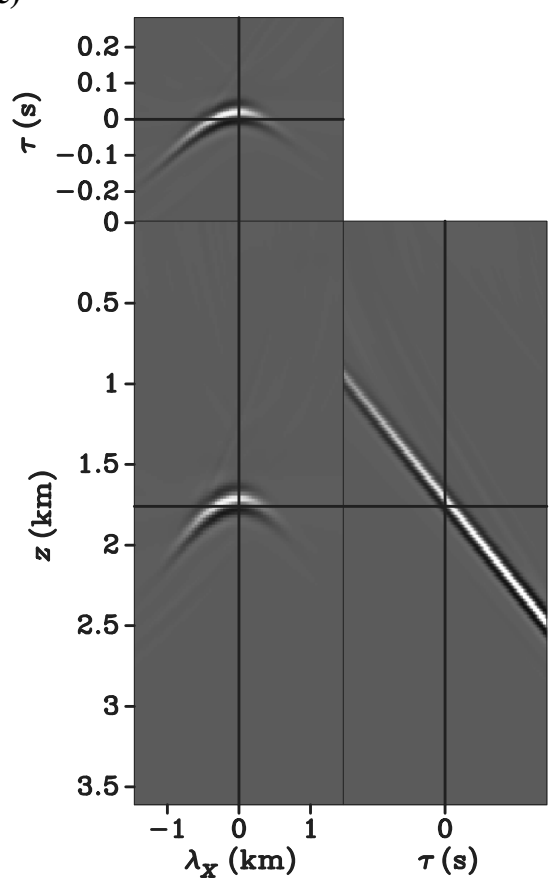

Figure 3. Space- and time-lag CIGs for model 1 computed with $\eta=0$ : (a) $x=1.5 \mathrm{~km}$, (b) $x=4.0 \mathrm{~km}$, and (c) $x=6.5 \mathrm{~km}$. Hereafter, the bottom-left and bottom-right panels display space-lag and time-lag extensions (respectively), whereas the top panels correspond to extended common-image-point gathers. 
scan over $\eta$ in the VTI layer from 0 to 0.3 with a 0.03 increment (the actual $\eta=0.15$ ), while using the actual $\delta=0.15$. For each value of $\eta$, we compute both time lags and inline horizontal space lags (Figures 3-5). The residual moveout in both space-lag CIGs and extended CIPs obtained for the dipping segments of the interface no longer has a "V" shape and resembles the residual caused by an inaccurate velocity model for isotropic media (Figures 3a, 3c, 4a, 4c, 5a, and 5c). This is explained by the fact that $\eta$ changes the NMO velocity and, therefore, conventional-spread moveout for dipping reflectors. Angle-domain CIGs for the dipping segments of the interface exhibit a)

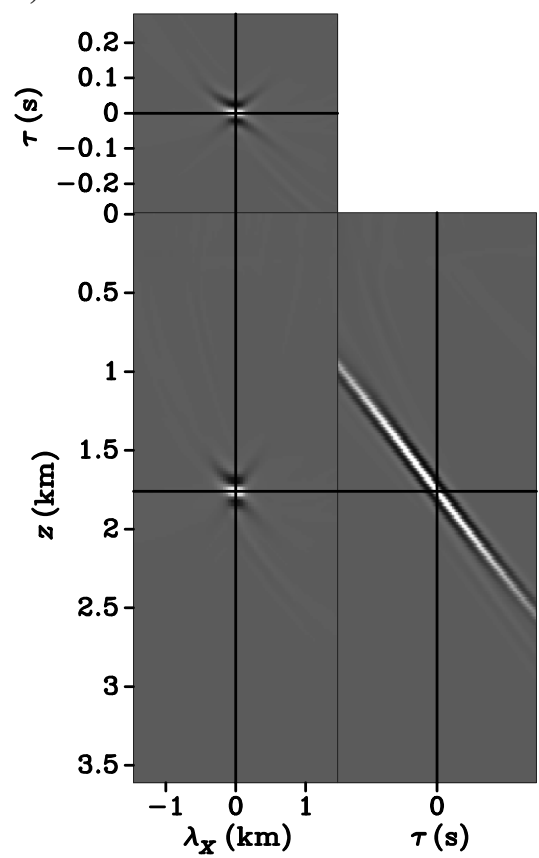

b)

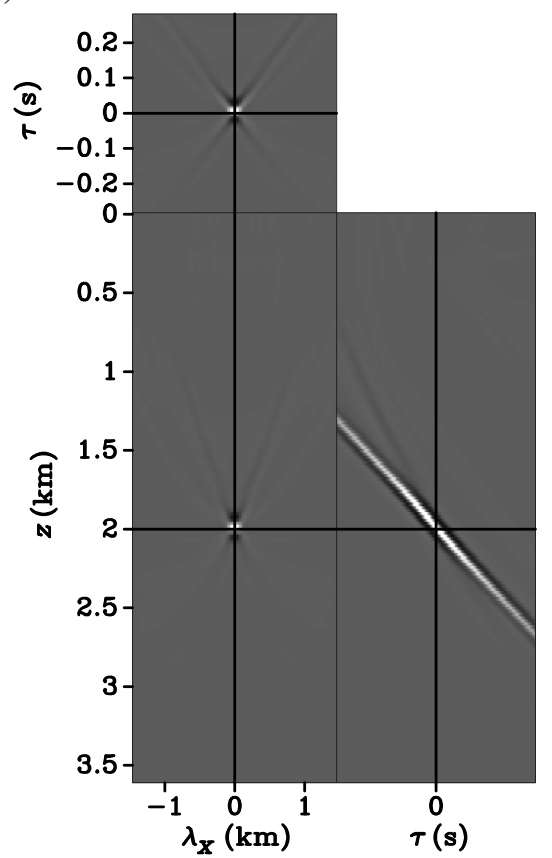

c)

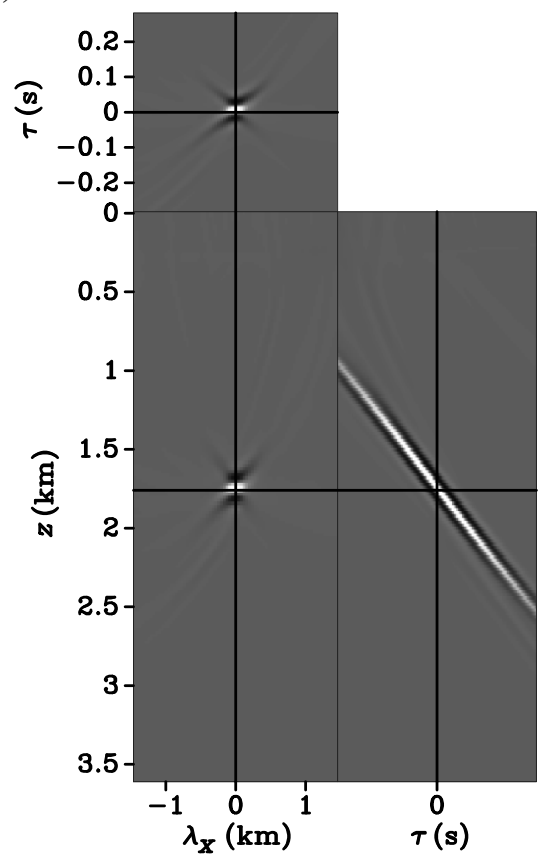

Figure 4. Space- and time-lag CIGs for model 1 computed with $\eta=0.15$ (actual value): (a) $x=1.5 \mathrm{~km}$, (b) $x=4.0 \mathrm{~km}$, and (c) $x=6.5 \mathrm{~km}$.

a)

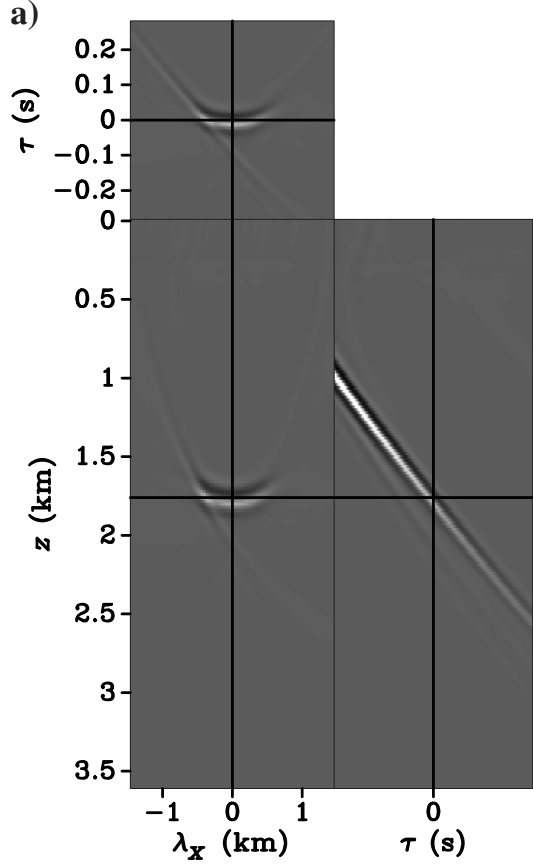

b)

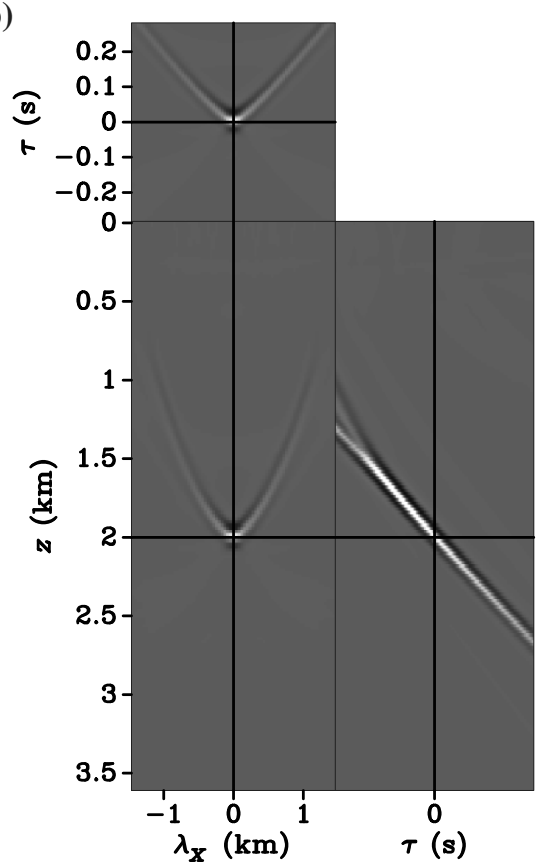

c)

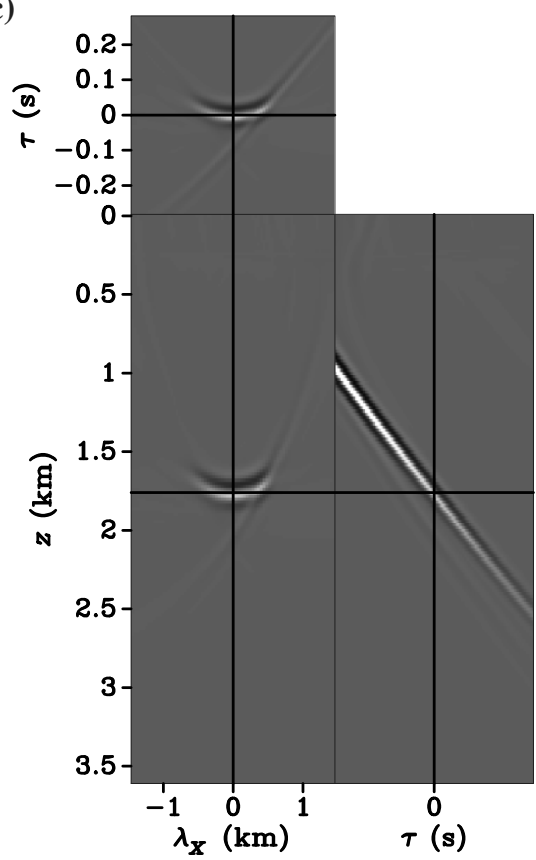

Figure 5. Space- and time-lag CIGs for model 1 computed with $\eta=0.3$ : (a) $x=1.5 \mathrm{~km}$, (b) $x=4.0 \mathrm{~km}$, and (c) $x=6.5 \mathrm{~km}$. 
a)

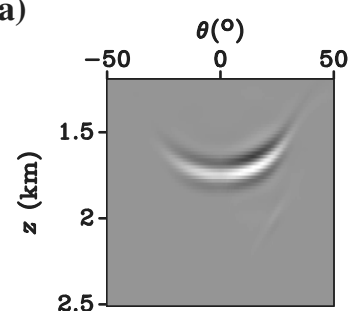

d)

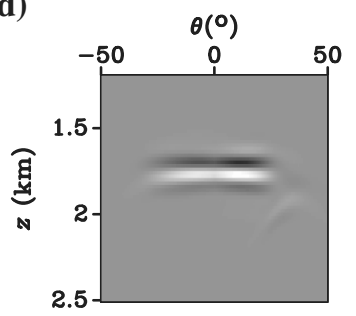

g)

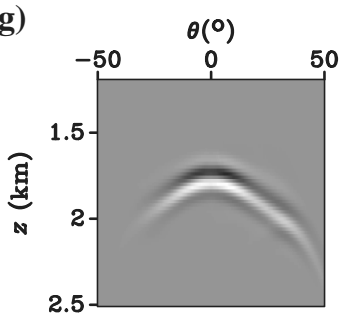

b)

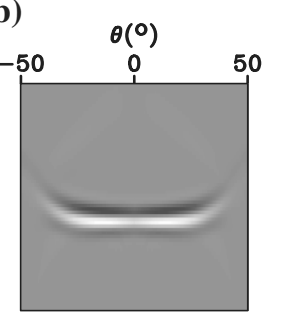

e)

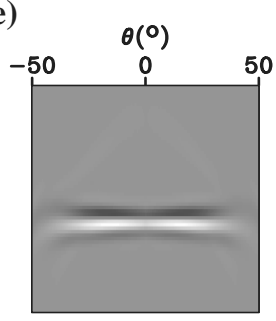

h)

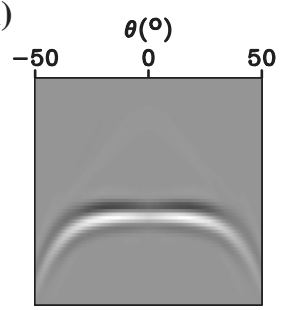

c)

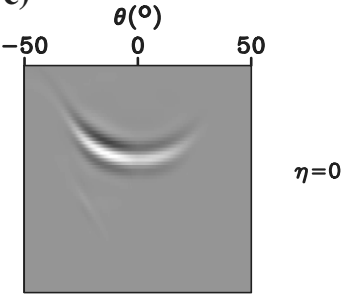

f)

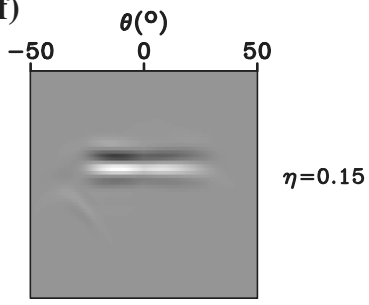

i)

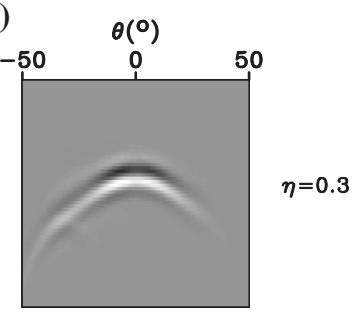

Figure 6. Angle-domain common-image gathers computed with $\eta=0$ (a, b, c), $\eta=0.15$ (actual value; $\mathrm{d}$, e, f), and $\eta=0.3(\mathrm{~g}, \mathrm{~h}, \mathrm{i})$ at $x=1.5 \mathrm{~km}(\mathrm{a}, \mathrm{d}, \mathrm{g})$, $x=4.0 \mathrm{~km}(\mathrm{~b}, \mathrm{e}, \mathrm{h})$, and $x=6.5 \mathrm{~km}(\mathrm{c}, \mathrm{f}, \mathrm{i})$.
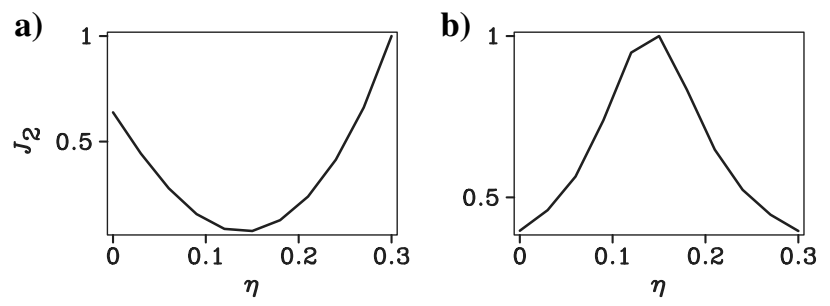

Figure 7. Influence of $\eta$ on the objective functions calculated using space-lag extended images for model 1: (a) DSO and (b) stackpower. residual moveout at all phase angles (Figure 6a and $6 \mathrm{c}$ ). In contrast, the RMO for the horizontal reflector segment is restricted to large incidence angles (Figure $6 \mathrm{~b}$ and $6 \mathrm{~h}$ ) because in the absence of dip $\eta$ influences only long-spread moveout.

The DSO and stack-power objective functions are shown in Figure 7. DSO produces an asymmetric function due to its inherent bias towards lower velocities (in this case, toward lower $\eta$ ). This bias can be explained by the fact that even for the actual $\eta$-field, extended images feature residual energy at nonzero lags due to the limited aperture. The presence of the residual energy distorts the DSO objective function. For model 1, imaging with an understated value of $\eta$ produces smaller residual energy compared to the one obtained with actual $\eta$, which shifts the minimum of the DSO objective function. To emphasize the dip-dependent influence of $\eta$, Figure 8 compares the DSO objective functions for the horizontal and dipping segments of the interface. Clearly, the energy focusing is more sensitive to the parameter $\eta$ in the presence of dip.

As expected, for this model $\delta$ does not influence the focusing in extended image gathers and the shape of objective functions, and only controls the depth of the imaged interface (Figure 9).

\section{Model 2}

Next, we use a modified segment of the BP 2007 tilted TI (TTI) model with an anticline structure (Figure 10). We simplify the model, which includes a tilted symmetry axis, as follows:

- The symmetry-axis tilt is removed to make the model VTI.

- The original $V_{\text {nmo }}$-field is smoothed, and only the two strongest reflectors are retained to avoid reflections from multiple interfaces.

- The parameter $\eta$ is taken to be constant $(\eta=0.15)$ throughout the model.

The spatially varying $\delta$-field in the original BP model is left unchanged. We obtain RTM extended images for values of $\eta$ ranging from 0 to 0.3 with a 0.05 increment. Due to the high computational cost, only the horizontal space lags are computed. As in the previous test, the signature of $\eta$ in space-lag CIGs generated with the actual $\delta$-field for the dipping interface segments deviates from the "V"-shape (Figure 11). The angle-domain CIGs show that the residual moveout caused a)

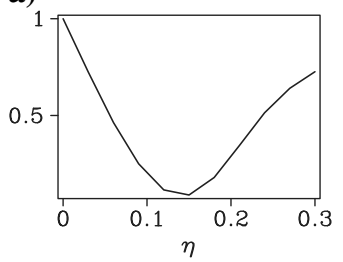

b)

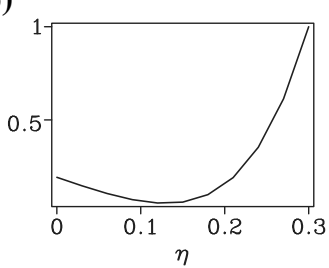

c)

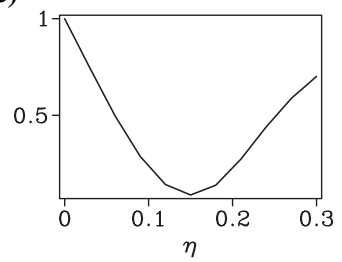

Figure 8. Influence of $\eta$ on the DSO objective function calculated using space-lag CIGs at: (a) $x=1.5 \mathrm{~km}$ (dipping segment), (b) $x=4.0 \mathrm{~km}$ (horizontal segment), and (c) $x=6.5 \mathrm{~km}$ (dipping segment). by errors in $\eta$ varies with dip (Figure 12). The DSO objective function has a minimum at the actual $\eta=0.15$, but is essentially flat between $\eta=0.1$ and 0.15 (Figure 13a).

Repeating the test with the erroneous $\delta=0$ shows that for subhorizontal reflector segments, the signature of $\eta$ maintains the "V"-shape even if $\delta$ is incorrect (Figure 14a, 14c, 14f, 14h, 14k, and $14 \mathrm{~m})$. As expected, the RMO in space-lag CIGs for dipping reflector segments due to an 
a)

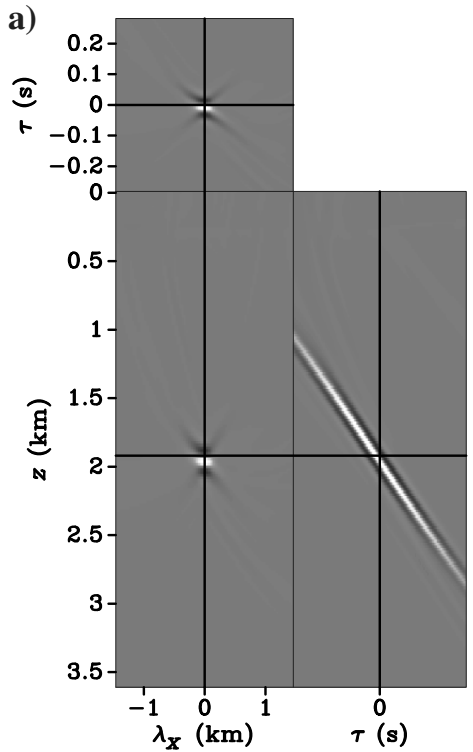

c)

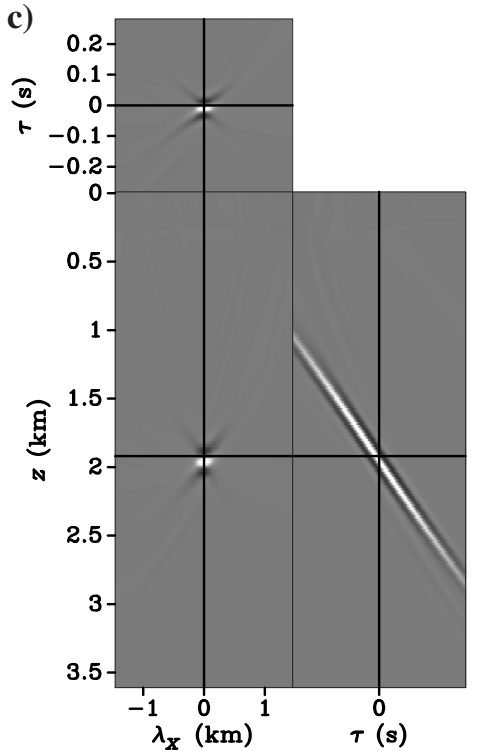

b)

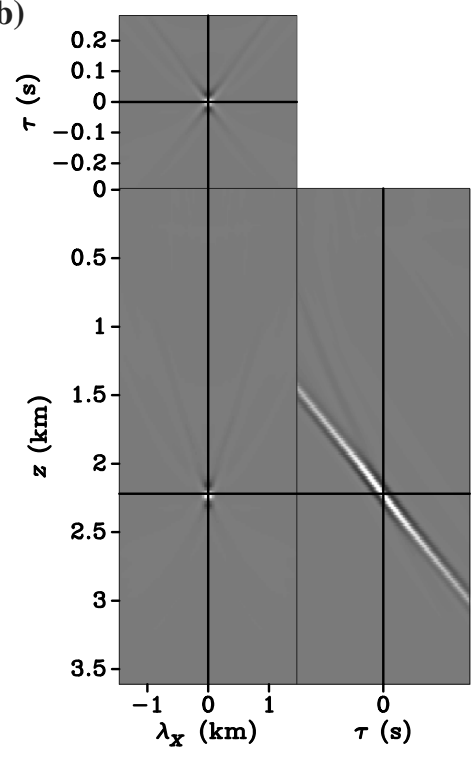

Figure 9. Images for model 1 obtained with the actual values of $V_{\text {nmo }}$ and $\eta$, but with $\delta=0$. Space- and time-lag CIGs at: (a) $x=1.5 \mathrm{~km}$, (b) $x=4.0 \mathrm{~km}$, and (c) $x=6.5 \mathrm{~km}$. (d) Conventional RTM image.

d)

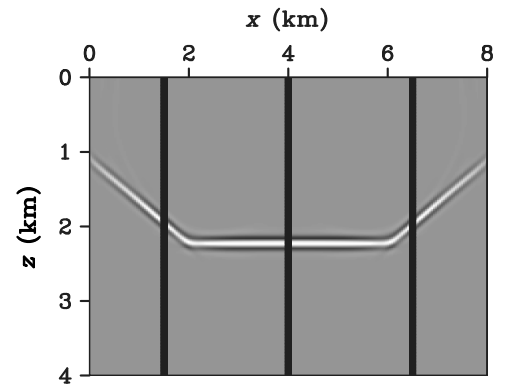

a)

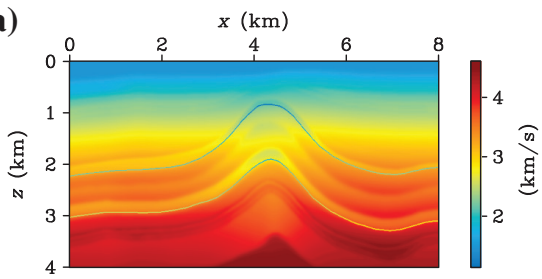

d)

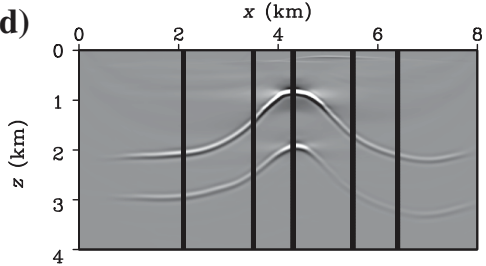

b)

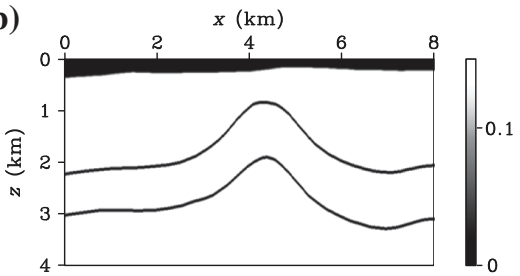

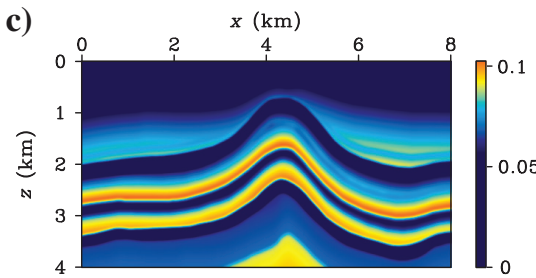

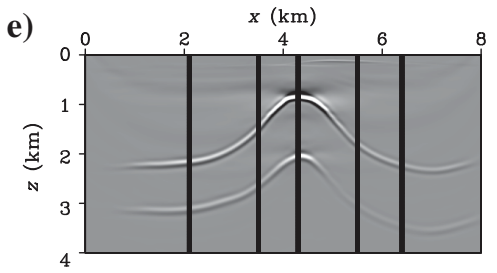

Figure 10. VTI model with an anticline structure (based on a section from the BP 2007 TTI model): (a) $V_{\text {nmo }}$, (b) $\eta$, and (c) $\delta$ (model 2), Conventional RTM image obtained (d) with the actual parameters and (e) with the actual $V_{\text {nmo }}$ and $\eta$, but $\delta=0$. The vertical lines mark locations of extended CIGs $(x=2.1,3.5,4.3,5.5,6.4 \mathrm{~km})$ used in the analysis. 
error in $\eta$ does not have the "V"-shape (Figure 14b, 14d, 14e, 14g, $14 \mathrm{i}, 14 \mathrm{j}, 14 \mathrm{l}, 14 \mathrm{n}$, and 14o). The corresponding angle gathers also illustrate the dip-dependent influence of $\eta$ (Figure 15). If $\delta$ is distorted, the extrema of the DSO and stack-power objective functions in Figure 16 are shifted toward lower $\eta$-values (close to 0.1 ).

Hence, the deviation of the $\eta$-signature from the "V"-shape for dipping interfaces that was identified in the previous example is also
Figure 11. Space-lag CIGs for model 2 computed with $\eta=0$ (a-e), $\eta=0.15$ (f-j), and $\eta=0.3$ (k-o). Each column corresponds to a fixed horizontal coordinate: $x=2.1 \mathrm{~km}(\mathrm{a}, \mathrm{f}, \mathrm{k}), x=3.5 \mathrm{~km} \mathrm{(b,g,}$ 1), $x=4.3 \mathrm{~km}(\mathrm{c}, \mathrm{h}, \mathrm{m}), x=5.5 \mathrm{~km}(\mathrm{~d}, \mathrm{i}, \mathrm{n})$, and $x=6.4 \mathrm{~km}(\mathrm{e}, \mathrm{j}, \mathrm{o})$.

Figure 12. Angle-domain common-image gathers for model 2 computed with $\eta=0$ (a-e), $\eta=0.15$ (f-j), and $\eta=0.3(\mathrm{k}-\mathrm{o})$. The horizontal coordinates for all five columns are the same as in Figure 11.

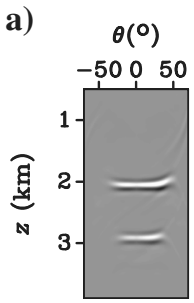

f)

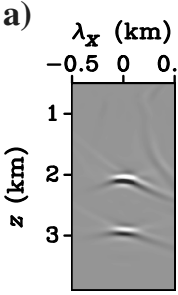

b) $\lambda_{x}(\mathrm{~km})$

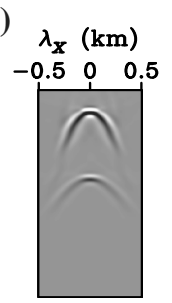

d) $\begin{array}{rl}\lambda_{x} & (\mathbf{k m}) \\ -0.5 & 0\end{array}$

e) $\lambda_{x}(\mathbf{k m})$

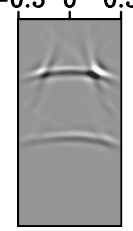

f)

$\lambda_{x}(\mathrm{~km})$

$\begin{array}{lll}-0.5 & 0 & 0.5\end{array}$

g)

$\lambda_{\mathrm{x}}(\mathrm{km})$

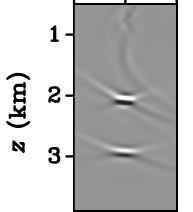

$\begin{array}{lll}-0.5 & 0 & 0.5\end{array}$

h)

k)

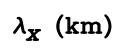

1)

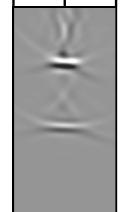

$\begin{array}{lll}-0.5 & 0 & 0.5\end{array}$

$\lambda_{x}(\mathrm{~km})$

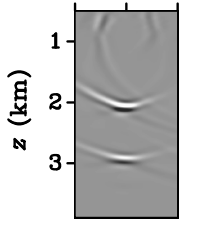

$\begin{array}{lll}-0.5 & 0 & 0.5\end{array}$

m)
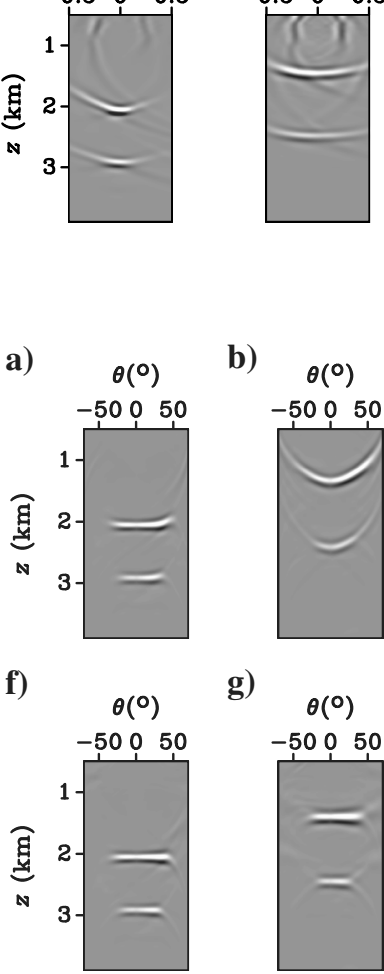

k)

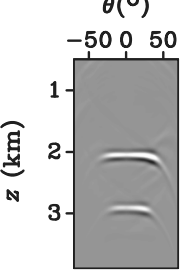

b)

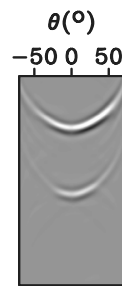

g)

c)

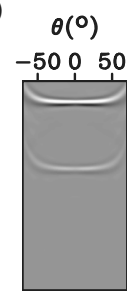

h)

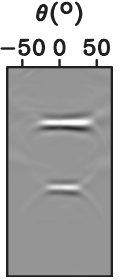

l)

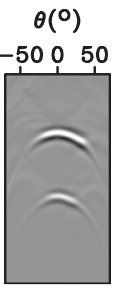

m)

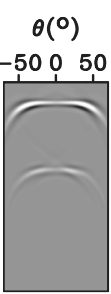

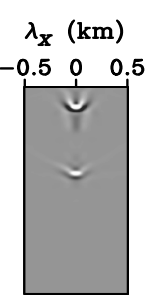
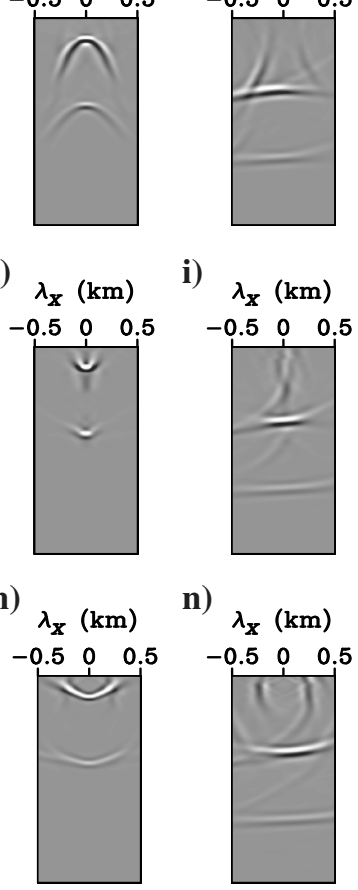

$\begin{array}{lll}-0.5 & 0 & 0.5\end{array}$

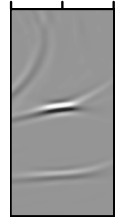

$\eta=0$

i)

$\begin{array}{rll}\lambda_{\mathbf{x}} & (\mathrm{km}) & \mathbf{j} \\ -0.5 & 0 & 0.5\end{array}$

$\lambda_{x}(\mathrm{~km})$

$\begin{array}{llllll}-0.5 & 0 & 0.5 & -0.5 & 0 & 0.5\end{array}$
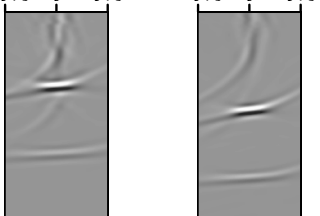

$\eta=0.15$

n)

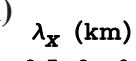

o)

$\begin{array}{lll}-0.5 & 0 & 0.5\end{array}$

$\lambda_{x}(\mathrm{~km})$

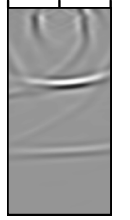

$\begin{array}{ll}0 & 0.5\end{array}$

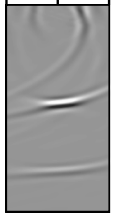

$\eta=0.3$

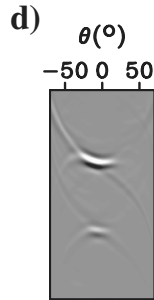

e)

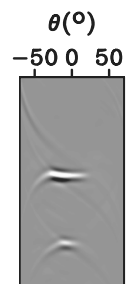

$\eta=0$

i)

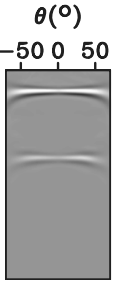

n)

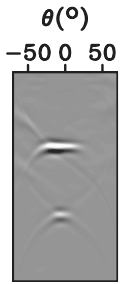

j)
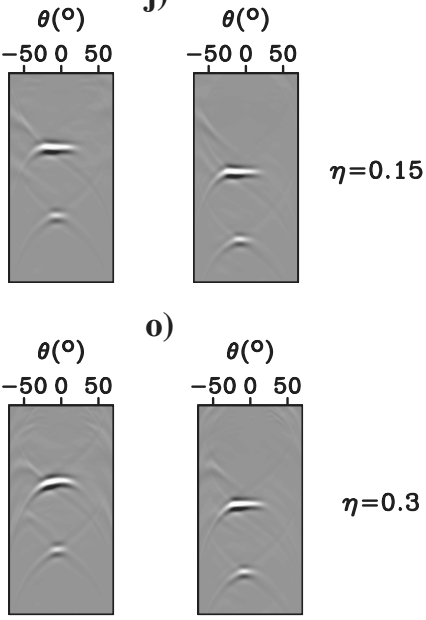

o)

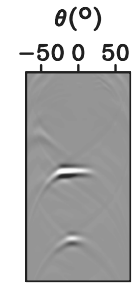

$\eta=0.3$ 
observed for this more complicated model. Also, the laterally varying $\delta$ influences the focusing in extended images, and, therefore, the shape of the $\eta$-dependent DSO and stack-power objective functions. However, because for model 2 the lateral change in $\delta$ is relatively mild, neglecting that parameter does not change the shape of RMO due to errors in $\eta$ for both subhorizontal and dipping interface segments.

\section{Model 3}

The last model includes pronounced Gaussian anomalies in $\eta$ and $\delta$ (reaching 0.15 at the center) embedded in a homogeneous isotropic background (Figure 17). We obtain extended images using the actual $\eta$-field and a variable magnitude of the $\delta$-anomaly (from 0 to 0.3 in 0.05 increments). Because of the large lateral variation in $\delta$, the distortions in that parameter not only cause inaccurate reflector positioning (Figure 18a and 18c) but influence the energy focusing. Space-lag extended CIGs in the middle of the model $(x=2.5 \mathrm{~km})$ exhibit noticeable defocusing for distorted peak values of $\delta$ (Figure 19a and 19c). Also, residual moveout in the angledomain CIGs is visible at all phase angles (Figure 19d and 19f).

The pronounced defocusing in the extended domain due to $\delta$-errors is explained by the fact that the large Gaussian anomaly in $\delta$ influences the NMO velocity. Because the reflector is horizontal, $\eta$ has a weaker influence on the reflection moveout since it contributes only to the nonhyperbolic moveout term. As shown above for model $1, \eta$-errors cause relatively weak defocusing for horizontal events. Moreover, since the anomaly in $\eta$ is located in the middle of the model, most far-offset rays do not even cross the anomaly.

The DSO objective function computed with the actual $\eta$-field has a well-defined minimum corresponding to the actual $\delta_{\max }$ (Figure 20a). The stack-power objective function, however, is biased towards larger $\delta_{\max }$-values (Figure 20b), which can be explained by the trade-off between geometric spreading and the defocusing due to errors in the velocity model. With increasing $\delta$, the source and receiver wavefields correlate at a smaller depth where they carry more energy.

In general, because DSO operates with amplitudes, the DSO objective function is influenced by such dynamic factors as the source radiation pattern, geometric spreading, reflection coefficient, and attenuation. Cross-correlation imaging condition does not take these factors into account and, therefore, RTM extended images obtained by cross-correlation do not provide the actual reflectivity distribution in the subsurface. As shown by, for example, Yang et al. (2013), Almomin and Biondi (2014), Hou and Symes (2015), and Lameloise et al. (2015), a more accurate estimate of the actual re-
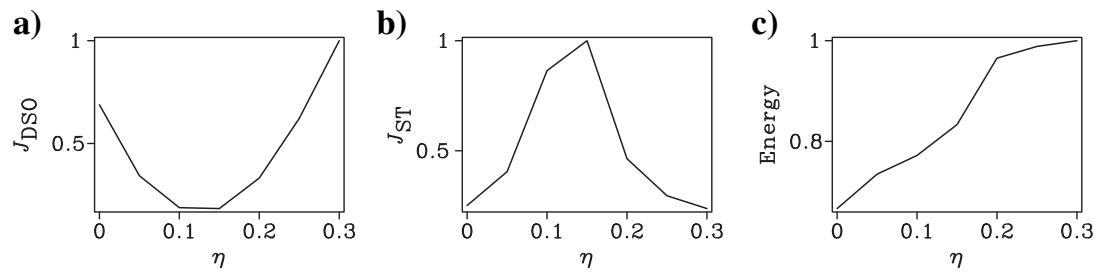

Figure 13. Influence of $\eta$ on the objective functions calculated from space-lag extended images obtained with the actual $\delta$-field for model 2: (a) DSO normalized by the energy of the extended image and (b) stack-power. (c) The energy of the extended image.

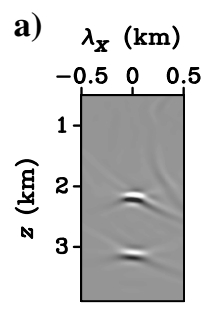

b) $\lambda_{x}(\mathrm{~km})$

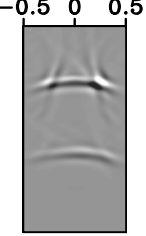

f)

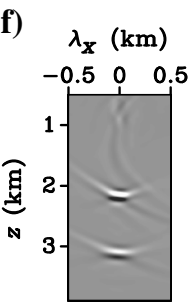

g)

$\lambda_{x}(\mathrm{~km})$
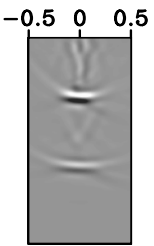

k)

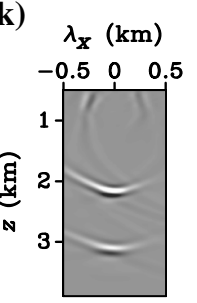

l)
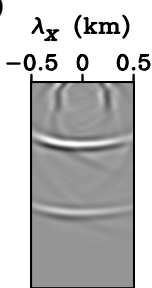

c) $\lambda_{x}(\mathrm{~km})$

$\begin{array}{lll}-0.5 & 0 & 0.5\end{array}$

d) $\lambda_{x}(\mathrm{~km})$

e) $\lambda_{x}(\mathrm{~km})$

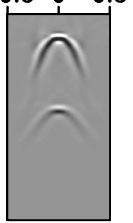

h)

h) $\lambda_{x}(\mathrm{~km})$
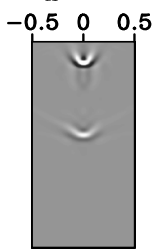

m)

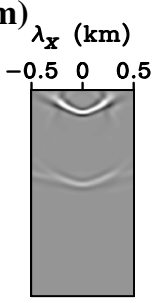

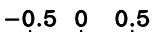

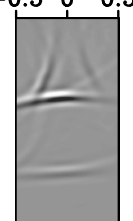

$\begin{array}{lll}-0.5 & 0 & 0.5\end{array}$

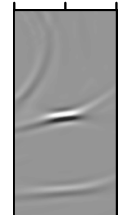

i)

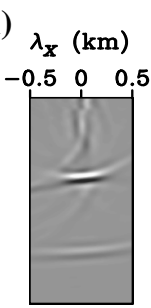

j)

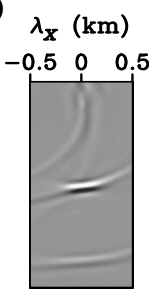

n)

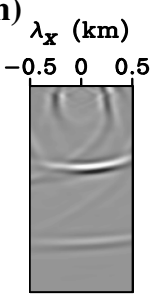

o)

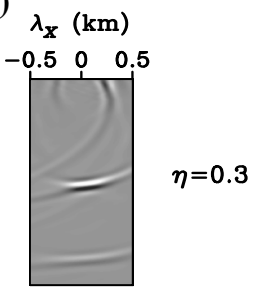

Figure 14. Space-lag CIGs for model 2 computed with $\delta=0$ and $\eta=0$ (a-e), $\eta=0.15$ (f-j), and $\eta=0.3(\mathrm{k}-\mathrm{o})$. The horizontal coordinates for all five columns are the same as in Figure 11. 
Figure 15. Angle-domain common-image gathers for model 2 computed with $\delta=0$ and $\eta=0$ (a-e), $\eta=0.15$ (f-j), and $\eta=0.3(\mathrm{k}-\mathrm{o})$. The horizontal coordinates for all five columns are the same as in Figure 11.

Figure 16. Influence of $\eta$ on the objective functions calculated from space-lag extended images obtained with $\delta=0$ for model 2: (a) DSO normalized by the energy of the extended image and (b) stack-power. (c) The energy of the extended image. a)

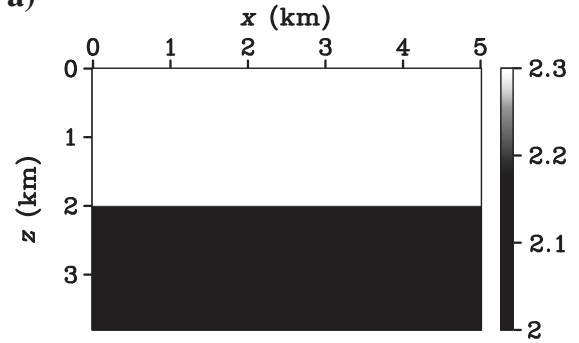

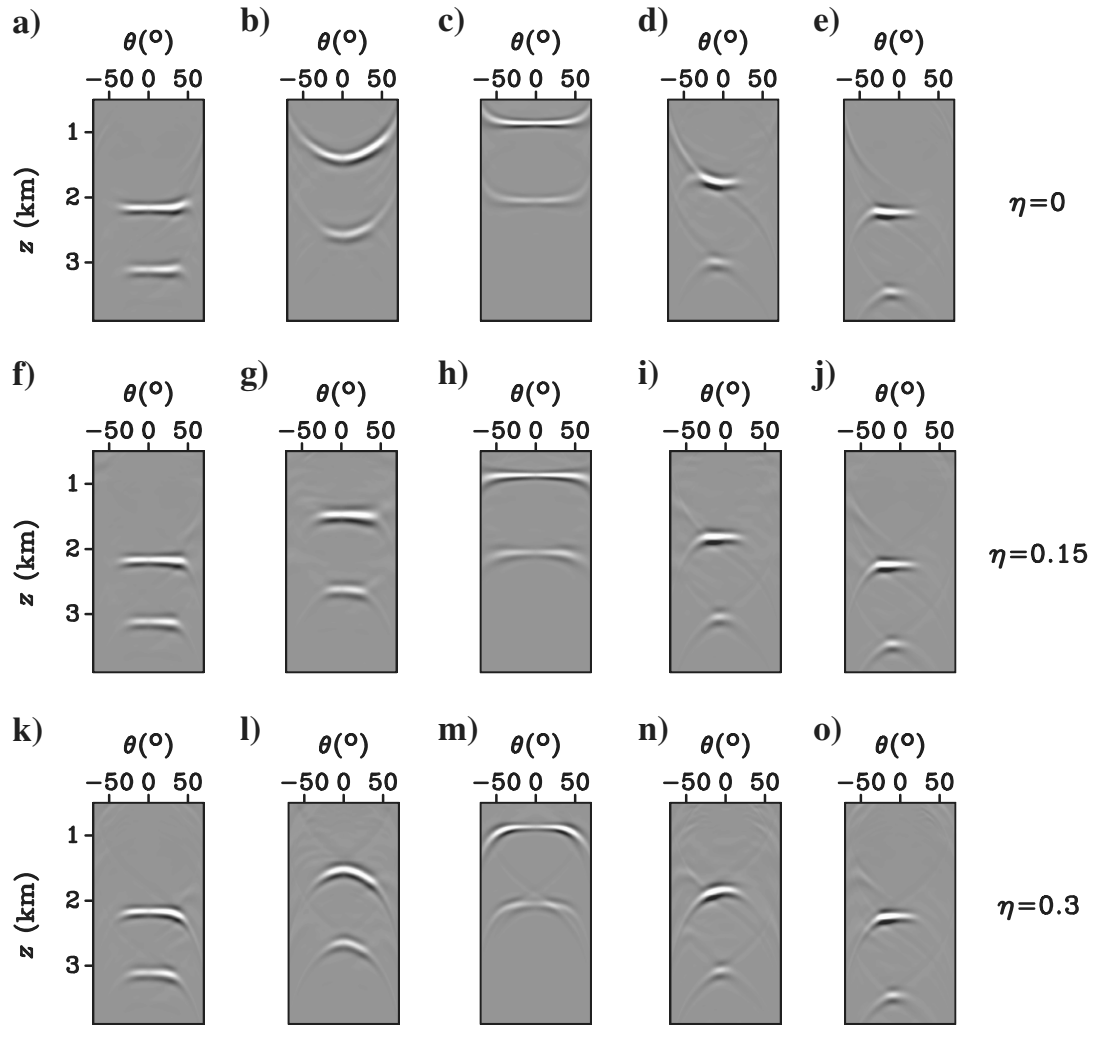
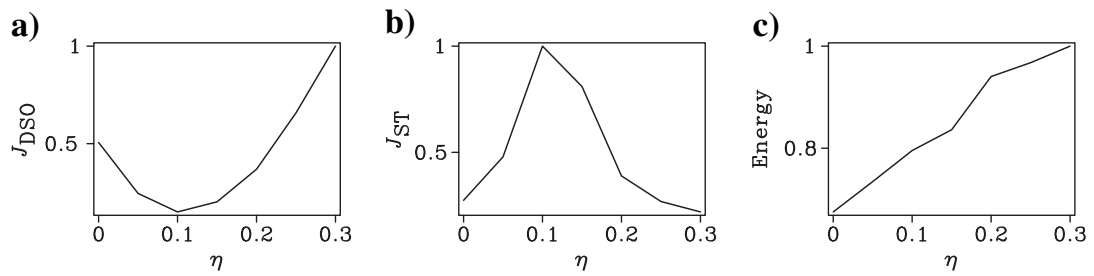

b)

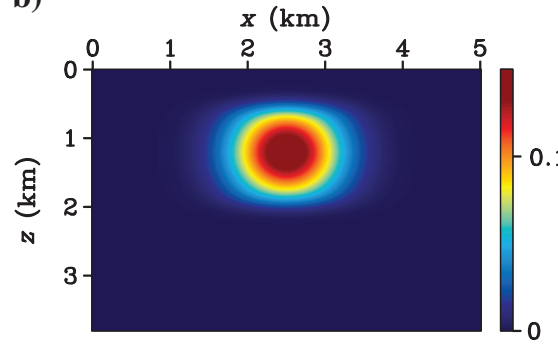

c)

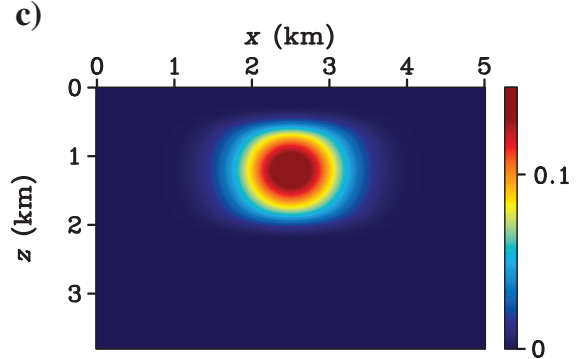

Figure 17. VTI model with identical Gaussian anomalies in $\delta$ and $\eta$ : (a) $V_{\text {nmo }}$, (b) $\eta$, and (c) $\delta$ (model 3).

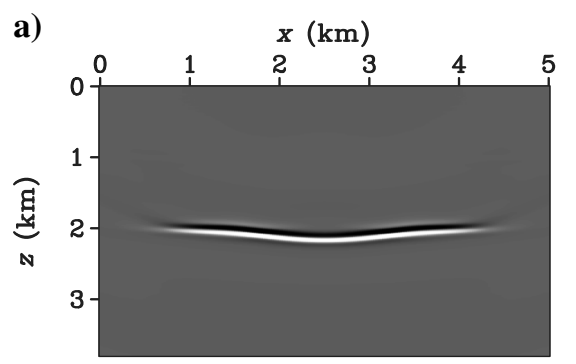

b)

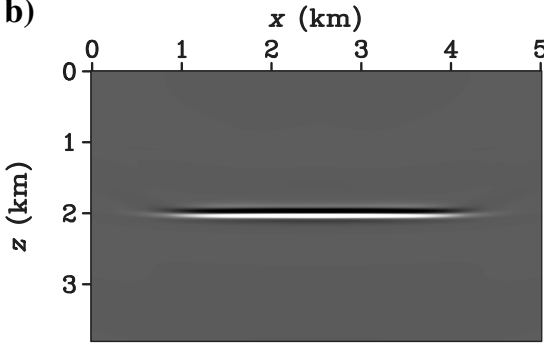

c)

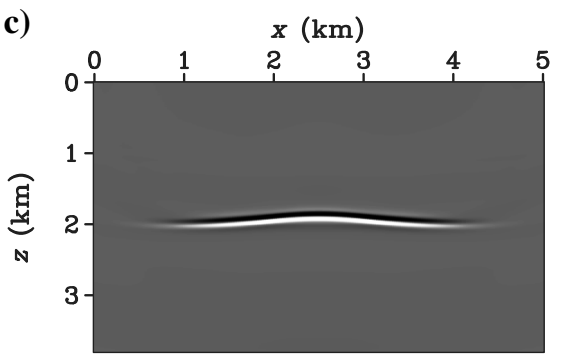

Figure 18. Conventional RTM images for model 3 computed with the actual $\eta$-field and different maximum values of $\delta$ : (a) $\delta_{\text {max }}=0$, (b) $\delta_{\max }=0.15$ (the actual value), and (c) $\delta_{\max }=0.3$. 


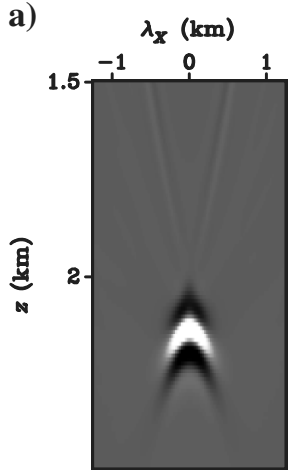

d)

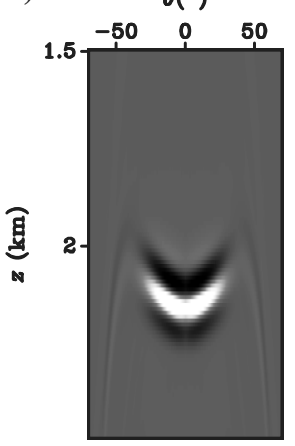

b)

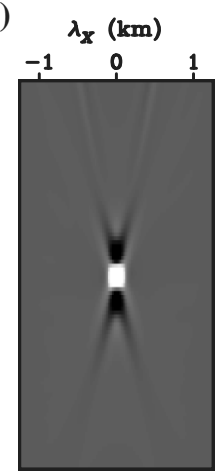

c)

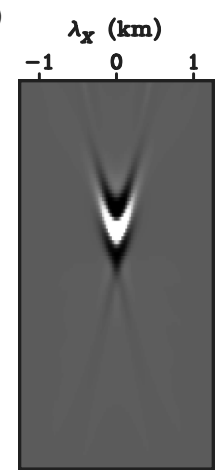

e)

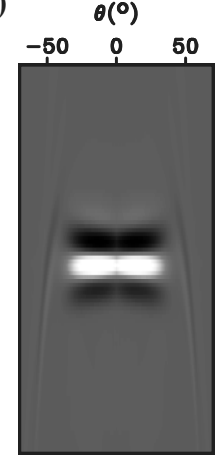

f)

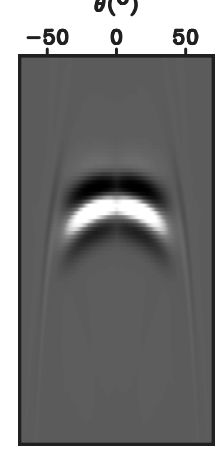

Figure 19. Space-lag (a, b, c) and angle-domain CIGs (d, e, f) for model 3 computed with the actual $\eta$-field and different maximum values of $\delta$ : (a, d) $\delta_{\max }=0,(\mathrm{~b}, \mathrm{e}) \delta_{\max }=0.15$ (actual value), and (c, f) $\delta_{\max }=0.3$.
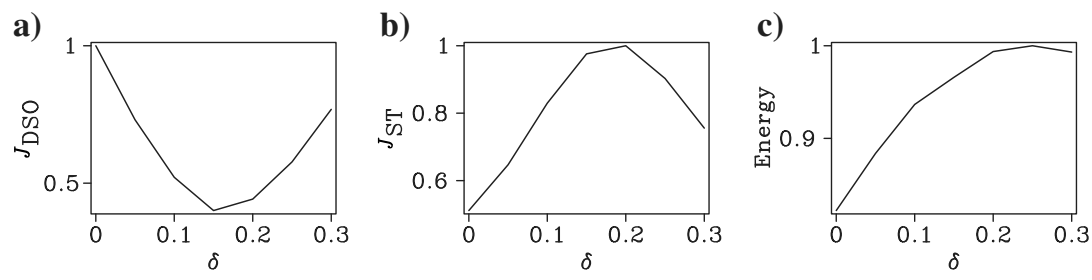

Figure 20. Influence of $\delta$ on the objective functions calculated from space-lag extended images obtained with the actual $\eta$-field for model 3: (a) DSO normalized by the energy of the extended image and (b) stack-power. (c) The energy of the extended image.

flectivity helps improve tomography algoritms that operate with extended images.

\section{CONCLUSIONS}

We presented a study of the anisotropy signature in RTM extended images for VTI models with dipping interfaces and laterally varying $V_{\text {nmo- }}$ and $\delta$-fields. The residual moveout due to errors in $\eta$ maintains a linear ("V"-like) shape for subhorizontal interfaces, regardless of the complexity of the overburden. If the reflector is dipping, errors in $\eta$ lead to more substantial defocusing in the extended image domain, and the shape of the residual moveout is similar to that caused by velocity distortions for isotropic media. The different signature of $\eta$ for dipping interfaces is explained by the influence of $\eta$ on NMO velocity, which becomes pronounced for dips reaching $25^{\circ}-30^{\circ}$. It should be emphasized that the deviation of the $\eta$-signa-

ture from the "V"-shape for dipping interfaces is observed even for a homogeneous overburden.

The DSO and stack-power objective functions demonstrate that the energy focusing in extended images is sensitive to the magnitude of the lateral variation of $\delta$. For a simplified segment of the BP TI model, accurate estimation of $\eta$ from either function requires including $\delta$ in the inversion. However, since the lateral variation in $\delta$ for the BP model is mild, setting $\delta=0$ does not visibly change the shape of residual moveout caused by errors in $\eta$.

For a model with significant $(0.15)$ Gaussian anomalies in $\eta$ and $\delta$, images obtained with inaccurate $\delta$-fields exhibit noticeable defocusing. The DSO objective function computed with the actual $\eta$-field has a well-defined minimum for the actual value of $\delta_{\max }$, which implies that for this model $\delta$ could potentially be constrained by P-wave reflection data.

Analysis of the sensitivity of the objective function to changes in the parameters is important in designing inversion algorithms in the extended image domain. This study provides insights into DSObased tomographic VTI inversion including the need for regularization in parameter estimation.

\section{ACKNOWLEDGMENTS}

We are grateful to Paul Sava (CWP) and members of the A(nisotropy) and (maging) teams at CWP for useful discussions. Model 2 represents a modified version of the TTI model created by Hemang Shah of BP (http://www.freeusp.org/2007_BP_Ani_Vel_ Benchmark/). This publication is based upon work supported by the Consortium Project on Seismic Inverse Methods for Complex Structures at CWP and the King Abdullah University of Science and Technology (KAUST) Office of Sponsored Research (OSR) under Award No. OCRF-2014CRG3-32140407/ORS\#2230. The reproducible numeric examples in this paper use the Madagascar open-source software package (Fomel et al., 2013a) freely available from http://www.ahay.org.

\section{REFERENCES}

Al-Yahya, K., 1989, Velocity analysis by iterative profile migration: Geophysics, 54, 718-729, doi: 10 $.1190 / 1.1442699$

Alkhalifah, T., 1996, Transformation to zero offset in transversely isotropic media: Geophysics, 61, 947963, doi: 10.1190/1.1444044.

Alkhalifah, T., 1997, Kinematics of 3-D DMO operators in transversely isotropic media: Geophysics, 62, 1214-1219, doi: 10 $.1190 / 1.1444222$.

Alkhalifah, T., 1998, Acoustic approximations for processing in transversely isotropic media: Geophysics, 63, 623-631, doi: 10.1190/1.1444361.

Alkhalifah, T., 2000, An acoustic wave equation for anisotropic media: Geophysics, 65, 1239-1250, doi: 10.1190/1.1444815.

Alkhalifah, T., and S. Fomel, 2011, The basic components of residual migration in VTI media using anisotropy continuation: Journal of Petroleum Exploration and Production Technology, 1, 17-22, doi: 10.1007/ s13202-011-0006-6.

Alkhalifah, T., S. Fomel, and B. Biondi, 2001, The space-time domain: Theory and modeling for anisotropic media: Geophysical Journal International, 144, 105-113, doi: 10.1046/j.1365-246x.2001.00300.x.

Alkhalifah, T., and I. Tsvankin, 1995, Velocity analysis for transversely isotropic media: Geophysics, 60, 1550-1566, doi: 10.1190/1.1443888.

Almomin, A., and B. Biondi, 2014, Preconditioned tomographic full waveform inversion by wavelength continuation: 84th Annual International Meeting, SEG, Expanded Abstracts, 944-948.

Anderson, J., T. Alkhalifah, and I. Tsvankin, 1996, Fowler DMO and time migration for transversely isotropic media: Geophysics, 61, 835-845, doi: 10.1190/1.1444008. 
Baysal, E., D. D. Kosloff, and J. W. C. Sherwood, 1983, Reverse time migration: Geophysics, 48, 1514-1524, doi: 10.1190/1.1441434.

Berkhout, A. J., 1982, Imaging of acoustic energy by wavefield extrapolation: Elsevier

Biondi, B., 2007, Angle-domain common-image gathers from anisotropic migration: Geophysics, 72, no. 2, S81-S91, doi: 10.1190/1.2430561.

Chavent, G., and C. A. Jacewitz, 1995, Determination of background velocities by multiple migration fitting: Geophysics, 60, 476-490, doi: 10 $.1190 / 1.1443785$.

Claerbout, J. F., 1985, Imaging the earth's interior: Blackwell Scientific Publications.

Cullison, T., and P. Sava, 2011, An image-guided method for automatically picking common-image-point gathers: 81st Annual International Meeting, SEG, Expanded Abstracts, 3908-3912.

Duveneck, E., and P. M. Bakker, 2011, Stable P-wave modeling for reversetime migration in tilted TI media: Geophysics, 76, no. 2, S65-S75, doi: 10 $1190 / 1.3533964$

Duveneck, E., P. Milcik, P.M. Bakker, and C. Perkins, 2008, Acoustic VTI wave equations and their application for anisotropic reverse-time migration: 78th Annual International Meeting, SEG, Expanded Abstracts, 2186-2190.

Fletcher, R. P., X. Du, and P. J. Fowler, 2009, Reverse time migration in tilted transversely isotropic (TTI) media: Geophysics, 74, no. 6, WCA179WCA187, doi: 10.1190/1.3269902.

Fomel, S., P. Sava, I. Vlad, Y. Liu, and V. Bashkardin, 2013a, Madagascar: Open-source software project for multidimensional data analysis and reproducible computational experiments: Journal of Open Research Software, 1, e8, doi: 10.5334/jors.ag.

Fomel, S., L. Ying, and X. Song, 2013b, Seismic wave extrapolation using lowrank symbol approximation: Geophysical Prospecting, 61, 526-536, doi: $10.1111 /$ j.1365-2478.2012.01064.x

Fowler, P. J., X. Du, and R. P. Fletcher, 2010, Coupled equations for reverse time migration in transversely isotropic media: Geophysics, $\mathbf{7 5}$, no. 1 , S11-S22, doi: 10.1190/1.3294572.

Grechka, V., L. Zhang, and I. James W. Rector, 2004, Shear waves in acoustic anisotropic media: Geophysics, 69, 576-582, doi: 10.1190/1.1707077.

Hou, J., and W. W. Symes, 2015, An approximate inverse to the extended Born modeling operator: Geophysics, 80, no. 6, R331-R349, doi: 10 $.1190 /$ geo2014-0592.1.

Lameloise, C.-A., H. Chauris, and M. Noble, 2015, Improving the gradient of the image-domain objective function using quantitative migration for a more robust migration velocity analysis: Geophysical Prospecting, 63, 391-404, doi: 10.1111/1365-2478.12195.

Le Stunff, Y., V. Grechka, and I. Tsvankin, 2001, Depth-domain velocity analysis in VTI media using surface P-wave data: Is it feasible?: Geophysics, 66, 897-903, doi: 10.1190/1.1444979.

Li, Y., B. Biondi, R. Clapp, and D. Nichols, 2014, Wave-equation migration velocity analysis for VTI models: Geophysics, 79, no. 3, WA59-WA68, doi: 10.1190/geo2013-0338.1.

McMechan, G. A., 1983, Migration by extrapolation of time-dependent boundary values: Geophysical Prospecting, 31, 413-420, doi: 10.1111/ j.1365-2478.1983.tb01060.x.

Rickett, J., and P. Sava, 2002, Offset and angle-domain common imagepoint gathers for shot-profile migration: Geophysics, 67, 883-889, doi: $10.1190 / 1.1484531$

Sattlegger, J. W., 1975, Migration velocity determination. Part I: Philosophy: Geophysics, 40, 1-5, doi: 10.1190/1.1440512.
Sava, P., and T. Alkhalifah, 2011, Wide-azimuth angle-domain imaging for anisotropic reverse-time migration: 81st Annual International Meeting, SEG, Expanded Abstracts, 3114-3119.

Sava, P., and T. Alkhalifah, 2012, Anisotropy signature in extended images from reverse-time migration: 82nd Annual International Meeting, SEG, Expanded Abstracts, doi: 10.1190/segam2012-0124.1.

Sava, P., and S. Fomel, 2006, Time-shift imaging condition in seismic migration: Geophysics, 71, no. 6, S209-S217, doi: 10.1190/1.2338824.

Sava, P., and S. J. Hill, 2009, Overview and classification of wavefield seismic imaging methods: The Leading Edge, 28, 170-183, doi: 10.1190/1 .3086052 .

Sava, P., and I. Vasconcelos, 2009, Efficient computation of extended images by wavefield-based migration: 79th Annual International Meeting, SEG, Expanded Abstracts, 2824-2828.

Sava, P., and I. Vasconcelos, 2011, Extended imaging conditions for waveequation migration: Geophysical Prospecting, 59, 35-55, doi: 10.1111/j .1365-2478.2010.00888.x.

Sava, P., and I. Vlad, 2011, Wide-azimuth angle gathers for wave-equation migration: Geophysics, 76, no. 3, S131-S141, doi: 10.1190/1.3560519.

Sava, P. C. and S. Fomel, 2003, Angle-domain common-image gathers by wavefield continuation methods: Geophysics, 68, 1065-1074, doi: 10 $.1190 / 1.1581078$.

Shen, P., and W. W. Symes, 2008, Automatic velocity analysis via shot profile migration: Geophysics, 73, no. 5, VE49-VE59, doi: 10.1190/1 .2972021 .

Shen, P., W. W. Symes, and C. C. Stolk, 2003, Differential semblance velocity analysis by wave-equation migration: 73rd Annual International Meeting, SEG, Expanded Abstracts, 2132-2135.

Soubaras, R., and B. Gratacos, 2007, Velocity model building by semblance maximization of modulated-shot gathers: Geophysics, 72, no. 5, U67U73, doi: 10.1190/1.2743612.

Symes, W.W., and J. J. Carazzone, 1991, Velocity inversion by differential semblance optimization: Geophysics, 56, 654-663, doi: 10.1190/1 .1443082 .

Tsvankin, I., 2012, Seismic signatures and analysis of reflection data in anisotropic media: SEG

Tsvankin, I., and V. Grechka, 2011, Seismology of azimuthally anisotropic media and seismic fracture characterization: SEG.

Weibull, W. W., and B. Arntsen, 2013, Automatic velocity analysis with reverse-time migration: Geophysics, 78, no. 4, S179-S192, doi: 10 $.1190 /$ geo2012-0064.1.

Weibull, W. W., and B. Arntsen, 2014, Anisotropic migration velocity analysis using reverse-time migration: Geophysics, 79, no. 1, R13-R25, doi: 10 $.1190 /$ geo2013-0108.1.

Yang, T., and P. Sava, 2011, Wave-equation migration velocity analysis with time-shift imaging: Geophysical Prospecting, 59, 635-650, doi: 10.1111/j 1365-2478.2011.00954.x

Yang, T., and P. Sava, 2015, Image-domain wavefield tomography with extended common-image-point gathers: Geophysical Prospecting, 63 1086-1096, doi: 10.1111/1365-2478.12204.

Yang, T., J. Shragge, and P. Sava, 2013, Illumination compensation for image-domain wavefield tomography: Geophysics, 78, no. 5, U65-U76, doi: 10.1190/geo2012-0278.1.

Zhang, Y., H. Zhang, and G. Zhang, 2011, A stable TTI reverse time migration and its implementation: Geophysics, 76, no. 3, WA3-WA11, doi: $10.1190 / 1.3554714$. 